ARCHIVO ESPAÑOL DE ARTE, LXXXVIII, 352

OCTUBRE-DICIEMBRE 2015, pp. 418-424

ISSN: 0004-0428, eISSN: 1988-8511

doi: 10.3989 /aearte.2015.27

\title{
"JOANNES STRADANUS FACIEBAT": UN DIBUJO PREPARATORIO \\ PARA EL GRABADO DE LA PALA DE ALTAR DE S. ANNA IN GIOLICA DI PRATO \\ EN LA COLECCIÓN DE LA BIBLIOTECA NACIONAL DE ESPAÑA
}

\author{
Luca Pezzuto
}

Universidad de Roma L'Aquila

\begin{abstract}
El dibujo que representa a Santa Ana Metterza con San Nicolás de Tolentino y San Agustín conservado en la Biblioteca Nacional de España es obra inédita del pintor flamenco Jan Var der Straet y una de las pruebas definitivas del cuadro existente en el altar mayor de la iglesia de Santa Anna in Giolica en Prato.

Palabras clave: Dibujo italiano; siglo XVI; Jan van der Straet; Johannes Stradanus: grabado flamenco; Theodor Galle; Philip Galle; S. Anna in Giolica.

\section{"JOANNES STRADANUS FACIEBAT": A PREPARATORY DRAWING FOR THE PRINT OF THE ALTARPIECE OF S. ANNA IN GIOLICA (PRATO) IN THE COLLECTION OF THE BIBLIOTECA NACIONAL OF SPAIN}

The Virgin and Child with Saint Anne, Saint Augustin and Saint Nicholas of Tolentino, an unpublished drawing in the Biblioteca Nacional of Spain is by Johannes Stradanus. It served as a model for the print based on the painting still preserved in the church of St. Anne in Giolica in Prato.

Key words: Italian drawing; $16^{\text {th }}$ century; Jan van der Straet; Johannes Stradanus: Flemish print; Theodor Galle; Philip Galle; S. Anna in Giolica.

El dibujo que representa a Santa Ana Metterza con san Nicolás de Tolentino y san Agustín conservado en la Biblioteca Nacional de España (fig. 1) ${ }^{1}$ entró a formar parte de la colección de la institución pública madrileña en 1867 , cuando todo el patrimonio gráfico del pintor, erudito, arqueólogo e iconólogo D. Valentín Carderera (1796-1880) -más de 1.800 obras- fue adquirido por el Estado ${ }^{2}$. En el verso del dibujo, hoy pegado a un soporte moderno, se podía leer una posible inscripción del propio Carderera en la cual se establecía que "este dibujo es de Giorgion de Castel Franco", y es posible que el erudito lo hubiese comprado, junto a muchos otros -entre los cuales había una interesante colección montada sobre un segundo soporte de papel romano de color sepia de finales del siglo XVII-, a un buen precio en el mercado italiano durante su estancia de nueve años en la Península (1822-1830)․ La atribución al pintor véneto continuó vigente para Ángel M.

\footnotetext{
${ }^{1}$ Madrid, Biblioteca Nacional de España, DIB/15/13/1 (referencia precisa B 7244). Se trata de un dibujo sobre papel agarbanzado oscuro: pluma, pincel, tina parda, aguada y toques de blanco; 204 x $182 \mathrm{~mm}$.

${ }^{2}$ Para un reconstrucción exhaustiva de los motivos de la constitución de la colección gráfica de la Biblioteca Nacional véase Santiago Páez, 1992: p. 117-150. Para una introducción sobre Carderera Coleccionista: Arana, 2009: p. $87-115$.

${ }^{3}$ Santiago Páez, 1992: p. 122-125.
} 
Barcia (1841-1927) en su fundamental Catálogo de la colección de dibujos originales de la Biblioteca Nacional, un texto de 1906, de gran importancia científica ya que no es sólo una de las primeras publicaciones respecto a catálogos de otras grandes colecciones europeas, sino que también el número de dibujos catalogados, más de diez mil, abarcan un marco cronológico de cinco siglos (del XV al XIX) incluyendo las principales corrientes artísticas ${ }^{4}$. Aunque no han sido publicadas, existen varias investigaciones llevadas a cabo por parte de muchos estudiosos internacionales de dibujo italiano. El dibujo ha tenido varias atribuciones que podemos observar en las notas manuscritas en el paspartú. Después de una primera e improbable relación con el pintor Cesare Nebbia, fue relacionado con la escuela boloñesa del siglo XVI por parte de Marzia Faietti que recientemente tuvo la hipótesis de que podría tratarse de una obra de Giovan Battista Ramenghi, conocido como Bagnacavallo el joven ${ }^{5}$.

Si analizamos detalladamente la estructura y el estilo de la composición podemos observar que se trata de otra escuela a pesar de la mala conservación de esta valiosa obra. Observando con minuciosidad, se aprecia la definición del rostro de santa Ana, tan soez, demacrado y ojeroso, y también los pliegues verticales y cilíndricos del manto que cubre la cabeza de la santa, y que, sólidos y casi paralelos, le caen de una manera nada natural sobre la espalda (fig. 1). Además se observa el gusto "tardo-manierista" en la colocación de las figuras que no guardan consonancia con la estructura arcaica, bloqueada y absolutamente simétrica; estos aspectos, que, como se verá, debieron trazar los orígenes no solo del gusto contrarreformista de la época, sino también de las peticiones específicas de los comitentes ligados a unos modelos precedentes.

En efecto, se puede pensar en los lugares donde la iconografía de santa Ana Metterza -"mi è terza", es decir, una antepasada de Cristo, es una derivación del vulgar florentino medieval- tuvo su mayor difusión en el Cinquecento.

En aquella época, este elemento en sí mismo hubiera haber podido situar en la Toscana (y en particular en el área florentina) el dibujo preparatorio. Uno de los ejemplos más cercanos podría ser uno de Giorgio Vasari, que en 1548 pinta para "il reverendo padre frate Mariotto da Castiglioni Aretino [...] una Nostra Donna col suo figliolo in collo et Santa Anna con quella et San Salvestro

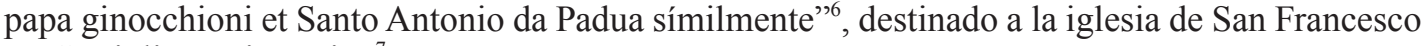
en Castiglione Fiorentino ${ }^{7}$.

Vasari a su vez debía tener en mente modelos de referencia de gran importancia, sobretodo la famosa Pala del Gran Consiglio de Fra Bartolomeo, hoy conservada en el Museo Nazionale di S. Marco en Florencia, con las diferentes versiones de los pintores florentinos de la primera mitad del Cinquecento.

Por tales razones, después de una investigación en el fondo de dibujos italianos del siglo XVI de la Biblioteca, me fue posible atribuir el dibujo al pintor flamenco Jan Van der Straet conocido como "Giovanni Stradano" y para ser más preciso clasificando el tema tratado que aparece en cuadro que representa a santa Anna Metterza entre san Agustín y Nicolás de Tolentino (que después fue sustituido por Filippo Benizzi), y que todavía hoy existe en el altar mayor de la iglesia de Santa Anna in Giolica en Prato (fig. 2). Pero para ser más exacto, el dibujo de Madrid debió ser un

\footnotetext{
${ }^{4}$ Barcia, 1906: inv. 7244.

${ }^{5}$ Para un resumen de las atribuciones, pero más en general, para tener información detallada del dibujo, véase la ficha del catálogo de la Biblioteca: signatura DIB/15/13/1, presente en la sección on-line de la colección en: $<$ http://catalogo.bne.es/uhtbn/cgisirsi/0jeCI71sT4/BNMADRID/240000448/123>.

${ }^{6}$ Giorgio Vasari, Ricordanze, (1527-1573), año 1548. Texto consultable on-line en la web de la Fondazione Memofonte en: <http://www.memofonte.it/home/files/pdf/vasari_ricordanze.pdf>.

${ }^{7}$ Para una profundización sobre la comisión de la pala de Castiglione Fiorentino, véase principalmente Reed, 1999: 678-681.
}

Arch. esp. arte, LXXXVIII, 352, OCTUBRE-DICIEMBRE 2015, 418-424

ISSN: 0004-0428, eISSN: 1988-8511, doi: 10.3989/aearte.2015.27 


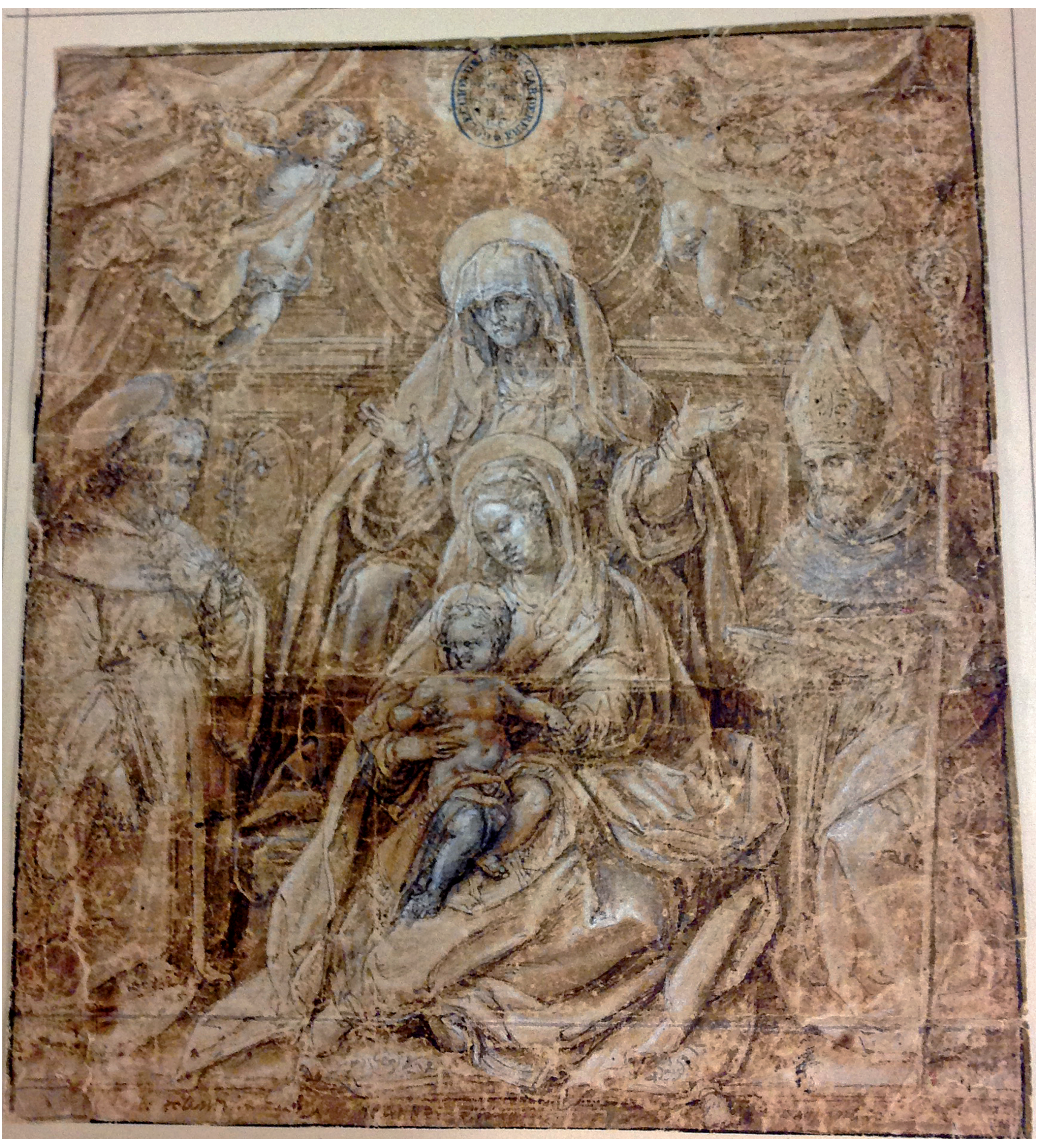

Fig. 1. Jan van der Straet, Santa Ana Metterza con San Nicolás de Tolentino y San Agustín, dibujo, Madrid, Biblioteca Nacional de España.

modelo utilizado después para la ejecución de un grabado de Theodor Galle (fig. 3) que propondría de nuevo el tema de la pintura toscana ${ }^{8}$.

Se trata de un dibujo sobre papel agarbanzado oscuro sobre el cual el artista, después de una primer esquicio a lápiz negro, traza los contornos a pluma y tinta parda y repasa la anatomía y las sombras de las figuras a pincel y tinta diluida y, al final, embellece los puntos de luz con el uso del albayalde, que desgraciadamente ha sufrido oxidaciones en bastantes partes del dibujo ${ }^{9}$. Este folio constituye el dibujo preparatorio, quizá fue el modelo presentado a la imprenta de Philip Galle dado el carácter tan acabado de la escena, la presencia de una inscripción ilegible en el

${ }^{8}$ Sobre Jan Van der Straet véanse principalmente: Gizzi, 1994; Baroni Vannucci, 1997; Baroni Vannucci/Sellink, 2012. Sobre la pintura de Prato se vean: Guasti, 1845-1860: 114; Marchini, 1956: 84; Fantappiè, 1983: 402-403; Baroni Vannucci, 1994: 88; 1997: 178-179 y Nardi, 2000: 128-132. Para mayor información sobre el grabado se puede ver la ficha presente en la sección on-line de la colección del British Museum con su correspondiente biblografía en: $<$ http://www.britishmuseum.org/research/collection_online/collection_object_details.aspx?objectId=1621353\&partId= $1 \&$ searchText=anne+galle\&page=1>. A esta se unen: Thiem, 1958:90 nota 8, que ha unido la estampa a un no bien especificado cuadro de Prato y el ensayo de Manfred Sellink en Baroni Vannucci/Seelink, 2012: 118-119 e 132 nota 27.

${ }^{9}$ El dibujo presenta grandes trazos retocados, probablemente de los posteriores dueños.

Arch. esp. arte, LXXXVIII, 352, OCTUBRE-DICIEMBRE 2015, 418-424 ISSN: 0004-0428, eISSN: 1988-8511, doi: 10.3989/aearte.2015.27 


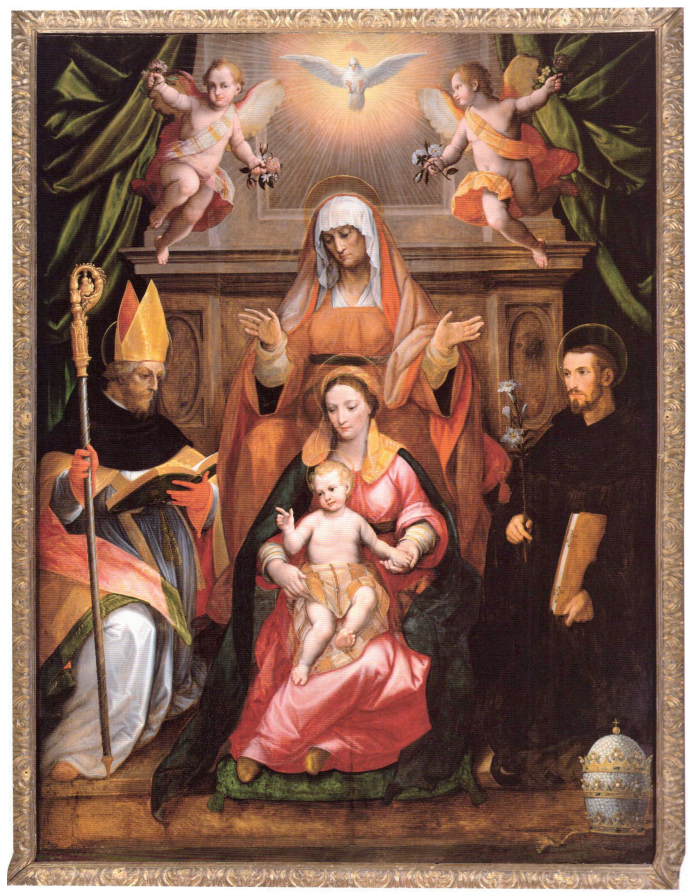

Fig. 2. Jan van der Straet, Santa Ana Metterza con San Nicolás de Tolentino y San Agustín, pintura, Prato, Iglesia di S. Anna in Giolica.

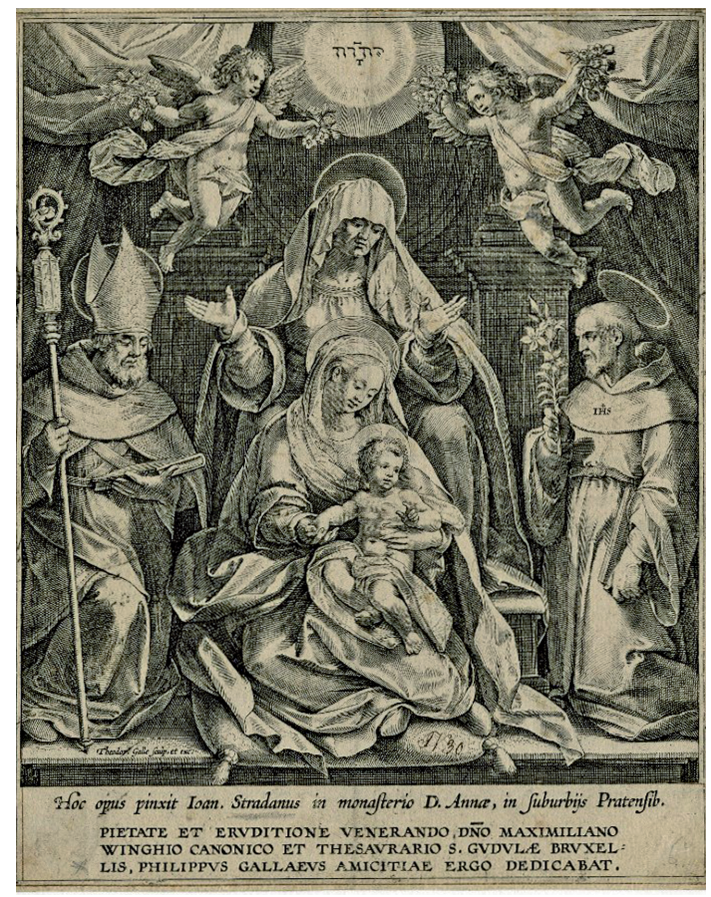

Fig. 3. Theodor Galle (por Jan van der Straet), Santa Ana Metterza con San Nicolás de Tolentino y San Agustín, grabado, London, British Museum.

margen inferior y la falta de arrepentimientos considerables ${ }^{10}$. Una característica de la práctica diseñadora de Stradano era que los dibujos preparatorios entregados a los grabadores con los que colaboraba tenían un nivel de acabado muy alto debido al uso de aguadas y albayalde ${ }^{11}$.

\section{La pintura}

La pala para la iglesia de Santa Ana en Giolica fue realizada en 1592 en un periodo de regreso de Flandes, después de su segunda experiencia napolitana y quizás también tenga relación el hecho de que mantuviese relaciones con el sector más culto de la corte de los Medici. Stradano se concentró principalmente en obras de carácter religioso, adaptándose a una comisión de literarios, eruditos y canónigos que estaban impregnados de las ideas del nuevo espíritu contrarreformista.

La pintura, firmada "Johannes Stradanus Faciebat 1592", ha tenido una historia complicada. En 1783 los frailes de la orden de los Servitas también llamados Siervos de Santa María se transfirieron al complejo agustiniano y a esta ocasión se debe la modificación de la iconografía a través

\footnotetext{
${ }^{10}$ Sobre la inscripción y sobre la relación entre Stradano y Galle se hablará más adelante.

${ }^{11}$ Sobre el tema, que se hablará más adelante, véanse Baroni Vannucci, 1997: 51-61 e Baroni Vannucci/Sellink, 2012 : 59-108.
}

Arch. esp. arte, LXXXVIII, 352, OCTUBRE-DICIEMBRE 2015, 418-424 ISSN: 0004-0428, eISSN: 1988-8511, doi: 10.3989/aearte.2015.27 
de la sustitución de san Nicolás de Tolentino con san Filippo Benizzi ${ }^{12}$. La fecha de ejecución hasta la última restauración fue interpretada como $1598^{13}$, a causa de la escasa legibilidad del friso, debida a un incendio que había dañado seriamente la obra ${ }^{14}$.

Si bien de la pala no se han encontrado noticias directas en los archivos, existen documentos que dicen que no se trata de la primera pintura de esta temática en el altar mayor de la iglesia ${ }^{15}$. Tal sucesión de encargos hacen creer que no solo las normas "post-tridentine" aún vigentes, o el nuevo espíritu devocional de Stradano, debieron determinar el arcaísmo "neo-quatrrocentesco" de la composición de época tardo cinquencentesca ${ }^{16}$, sino también que sin duda influyeron las peticiones de los comitentes muy ligadas a ciertos prototipos del siglo precedente. La sustitución de la pintura precedente con la nueva obra debía seguir recordando una tradición de culto bien arraigada en el área florentina, que podía recordar hasta los modelos más notables como el de Masaccio (pero de antes también), pasando por Fra Bartolomeo, los pintores de la escuela de S. Marco y de la Annunziata, hasta Vasari.

\section{El grabado}

En 1570 comenzó una colaboración con la imprenta Aux Quatre Vents de Hieronimus Cock, uno de los más importantes grabadores de Flandes, que representó una experiencia remunerativa y gratificante para Stradano ${ }^{17}$. A partir de la década sucesiva el artista flamenco se dedicó con mayor intensidad a la difusión a través del grabado de muchas de sus obras recientes: palas religiosas, tapices, grabados para libros y decoraciones efímeras. Fue con Philip Galle cuando Jan Van der Straet acordó entorno a 1580 un contrato propio para la realización de los grabados de sus dibujos, realizados al principio por el propio Galle y sucesivamente por su hijo Theodor, Heindrick Goltzius, Adrien Collaert e Hieronimus Wierix ${ }^{18}$, confirmado también en un pasaje de Raffaello Borghini:

"Cresciutogli al fine maggiormente l'animo ha fatto sei libri di disegni, che tuttavia s'intagliano in Anversa per mano di Filippo Gale eccellente intagliatore"" .

\footnotetext{
${ }^{12}$ Específicamente, la intervención pictórica consiste en la adición de la corona pontificia a los pies del santo agustiniano. El primer estudioso que vio esta modificación settecentesca fue Gaetano Guasti (Guasti, 1845: 117). Se ha creído que podía tratarse de Bernardo (Thiem, 1958: 90; Baroni 1997: 178); en cambio, después de la restauración de la pala, el personaje ha sido reconocido como Nicolás de Tolentino (Nardini, 2000: 128-132), identificación legitimada también en el dibujo madrileño y en el grabado de Galle.

${ }^{13}$ Guasti, 1845: 117; Baroni Vannucci, 1997: 178.

${ }^{14}$ Lo que queda de la inscripción original aparece en el borde inferior de la pintura. Después de una restauración se ha podido reconocer el año 1592 (Nardini 2000: 128-132).

${ }^{15}$ En las memorias manuscritas del convento, redactadas entorno a 1563, se puede leer: "Burnetto fece dipingere la tavola di S. Anna per l'Altar Maggiore, la quale vi stette per tutto l'anno 1349 al tempo di frate Zaccheria di Simone da Firenze, dal quale fu fatta dipingere la tavola che è oggi all'Altar Grande qual fu nell'anno 1487 riquadrata da Matteo Lachi nobil gentil uomo fiorentno mentre ch'era priore Fra Girolamo da Siena", Memorie diverse intorno al conento di Sant'Anna e al Beato Brunetto raccolte dal canonico Luigi Sacchi, Biblioteca Roncioniana di Prato, BRP, MS. 138, papel sin numeración. Texto citado por primera vez en Nardini, 2000: 130.

${ }^{16}$ Baroni Vannucci: 1994: 87.

${ }^{17}$ Las relaciones entre Stradano y el mundo del grabado se encuentran sobre todo en: Baroni, 1997: 61-68; Stijnman, 2010: 11-29; Baroni Vannucci/Sellink, 2012: 109-182.

${ }_{18}$ Baroni Vannucci/Sellink, 2012: 109-133.

${ }^{19}$ Borghini, Il Riposo, 1584: 42. Giorgio Vasari, Ricordanzae, (1527-1573), año 1548. Texto consultable on-line en el sitio web de la Fondazione Memofonte.
} 
Los dibujos que nos han llegado, en muchos casos, podrían ser dibujos preparatorios derivados de sus propias pinturas. En tales ocasiones, en los grabados venía a menudo mencionado el lugar y el tema que reproducía la estampa. Entra dentro de su producción el buril firmado "Theodor Galle sculp." (fig. 3), basado en su inédito dibujo de Madrid (fig. 1) y que representa el tema de la pala de altar de Sant'Anna in Giolica (fig. 2) ${ }^{20}$. La correspondencia de las dimensiones entre dibujo y grabado y la representación del tema confirma la relación aquí propuesta ${ }^{21}$. La presencia en el margen inferior del dibujo de Madrid de una inscripción ya citada con anterioridad, se puede leer "IOANNES STR" en algunas partes bien conservadas. Pero habría bastado con la comparación técnica y estilística entre el dibujo y la Madonna con il Bambino e Sant'Anna conservada en el Anton Ulrich Museum de Braunschweig para demostrar la validez de la atribución. Se trata de otro dibujo preparatorio de Stradano utilizado para otro grabado publicado por Philip Galle en la misma época, que se puede comparar con el dibujo español tanto por la ejecucción de los paños y en mayor medida por la estructura y fisionomía de los persoajes, especialmente con la figura de Santa Ana ${ }^{22}$.

Volviendo al buril que deriva del cuadro de Prato nos lleva a la siguiente inscripción: "hoc opus pinxit Ioan. Stradanus in monasterio D. Annae in suburbijs Pratensibus" y "pietate et eruditione venerando domino Maximiliano winghio canonico et thesaurario s. Gudulae bruxellis Philippus Gallaeus amicitiae ergo dedicabat". Por una parte, identifica el lugar donde se encuentra la pintura original, y por otra parte, la dedicación de la obra a Maximilian Winghius, canónigo y tesorero de la iglesia de Santa Gúdula en Bruselas, descendiente de una familia patricia bien conocida en la ciudad belga.

En estas pocas líneas se ha tratado de recrear una relación perdida entre tres objetos artísticos que en el pasado debieron estar conectados en el interior de una dinámica de producción y promoción de las imágenes de Jan Van der Straet y Philip Galle: el dibujo ejecutado por Stradano, la pintura de Prato y el grabado que fue tomado de Theodor Galle. El trámite para la circulación de la composición en el ámbito europeo fue el dibujo de Madrid, entregado por el pintor flamenco, junto a otros, a la imprenta de Galle. Un testimonio inédito que contribuye a la producción de grabados como medio divulgativo por excelencia de la segunda mitad del Cinquecento; una aportación para la reconstrucción de las relaciones culturales entre Italia y Flandes; así como un importante descubrimiento para la Biblioteca Nacional de España, donde en su colección, por mérito de la habilidad de Connousseur de Carderera ${ }^{23}$, hay un dibujo de Jan Van der Straet.

\section{AGRADECIMIENTOS}

Por el breve periodo pasado investigando en la Biblioteca Nacional, deseo agradecer de corazón a la Dr. Isabel García-Toraño Martínez, que me acogió espléndidamente en la institución facilitando del mayor modo posible mi trabajo. Agradezco también a la Dra. Elena Santiago Páez, sin su interés este artículo no habría existido, al director de la revista Wilfredo Rincón García por la oportunidad de presentar el artículo, al Dr. Paolo Benassai por haber hecho posible la visita a la iglesia de S. Anna in Giolica, a la Dra. Alessandra Baroni Vannucci, una de las expertas en Stradano, con la que he razonado muchas de las cuestiones. Agradezco sinceramente a la Dra. Noelia Muñoz Esteban su trabajo de traducción.

${ }^{20}$ Londres, British Museum, 1874,0110.444 (Museum number). Buril, 230 x 184 mm. El grabado del British Museum es un segundo estado de dos. Para mayor información sobre el buril véase Leesberg 2008: 92-93.

${ }^{21}$ Las dimensiones del dibujo y del grabado mencionado coinciden $(204 \times 182 \mathrm{~mm}$ per il foglio spagnolo e $230 \mathrm{x}$ $184 \mathrm{~mm}$ para el buril). La variación en altura de cerca de $26 \mathrm{~mm}$ representa el espacio de la inscripción inferior. Para las referencias bibliográficas véase nota 8 .

${ }^{22}$ Para la ilustración y mayor información sobre el dibujo se vea Baroni 1997: n. 542.

${ }^{23}$ El cual, no consiguió atribuir el dibujo (lo restituyó a Giorgione), comprendió la alta cualidad, decidiendo adquirirlo para su colección.

Arch. esp. arte, LXXXVIII, 352, oCTUBRE-DICIEMBRE 2015, 418-424 ISSN: 0004-0428, eISSN: 1988-8511, doi: 10.3989/aearte.2015.27 


\section{BIBLIOGRAFÍA}

Arana, Itziar (2009): "Valentín Carderera, comisionado, académico y coleccionista“. En: Socias Batet, Immaculata/Aguilar, Miriam (eds.) (2009): Conflictes bèllics, espoliacions, colleccions. Barcelona: Edicions de la Universidad de Barcelona, pp. 87-115.

Barcia, Angel María (1906): Catálogo de la colección de dibujos originales de la Biblioteca Nacional. Madrid: De La Cuesta.

Baroni Vannucci, Alessandra (1994): “Giovanni Stradano pittore e disegnatore, note e appunti sull'attività del Fiammingo dal 1574 all'anno di morte 1605”. En: Gizzi, Corrado (eds.) (1994): Giovanni Stradano e Dante (Torre de' Passeri (PE), Casa Dante in Abruzzo, 01/10 - 30/11 1994). Milán: Electa, pp. 79-90.

Baroni Vannucci, Alessandra (1997): Jan van der Straet detto Giovanni Stradano. Milán: Sapi.

Baroni Vannucci, Alessandra/Sellink, Manfred (eds.) (2012): Stradanus, 1523-1605, court artist of the Medici (Groeningemuseum, Bruges, 09/10/2008-04/01/2009). Turnhout: Brepols.

Fantappiè, Renzo (1983): "Schede di tesori d'arte di Prato con illustrazioni iconografiche e documentarie". En: Fantappiè, Renzo: Il bel Prato. Prato: Edizioni del Prato, vol. II.

Gizzi, Corrado (1994): Giovanni Stradano e Dante (Torre de' Passeri (PE), Casa Dante in Abruzzo, 01/1030/11 1994). Milán: Electa

Guasti, Gaetano (1845-1848): Pel calendario pratese, memorie e studi di cose varie. Prato: Guasti.

Leesberg, Marjolein (2008): The New Hollstein Dutch \& Flemish Etchings, Engravings and Woodcuts 14501700. Johannes Stradanus, part II. Ámsterdam: Sound \& Vision Pubblishers.

Marchini, Giuseppe (1956): Prato, guida artistica. Florencia: Arnaud.

Nardi, Cristina (2000): Sant'Anna in Giolica. Prato: C. Mantini.

Reed, Richard (1999): "Vasari's altar-piece at Castiglione Fiorentino". En: The Burlington Magazine, 141, 1, Londres, pp. 678-681.

Santiago Páez, Elena (1992): "Los fondos del Servicio de Dibujos y Grabados de la Biblioteca Nacional". En: Boletín de la Asociación de Archiveros, Bibliotecarios, Museólogos y Documentalistas, XLII, 1, Madrid, pp.117-150.

Stijnman, Ad (2010): “Stradanus's print shop”. En: Print quarterly, 27, 1, Londres, pp. 11-29.

Thiem, Gunther (1958): "Studien zu Jan van der Straet, genannt Stradanus. I Stradanus als Zeichner". En: Mitteilungen des Kunsthistorischen Institutes in Florenz, 8, 2, Florencia, pp. 88-111.

Fecha de recepción: 1-XII-2014

Fecha de aceptación: 9-II-2015

Arch. esp. arte, LXXXVIII, 352, OCTUBRE-DICIEMBRE 2015, 418-424 ISSN: 0004-0428, eISSN: 1988-8511, doi: 10.3989/aearte.2015.27 